\title{
UNIVERSITYOF
}

FORWARD

THINKING

WESTMINSTER用

WestminsterResearch

http://www.westminster.ac.uk/westminsterresearch

Physical fitness and prior physical activity are both associated with less cortisol secretion during psychosocial stress

Wood, C., Clow, A., Hucklebridge, F., Law, R. and Smyth, N.

This is an accepted manuscript of an article published by Taylor \& Francis in Anxiety, Stress and Coping, 31 (2), pp. 135-145.

The final definitive version is available online:

https://dx.doi.org/10.1080/10615806.2017.1390083

(C) 2018 Taylor \& Francis

The WestminsterResearch online digital archive at the University of Westminster aims to make the research output of the University available to a wider audience. Copyright and Moral Rights remain with the authors and/or copyright owners.

Whilst further distribution of specific materials from within this archive is forbidden, you may freely distribute the URL of WestminsterResearch: ((http://westminsterresearch.wmin.ac.uk/).

In case of abuse or copyright appearing without permission e-mail repository@westminster.ac.uk 


\section{Physical fitness and prior physical activity are both associated with less cortisol}

secretion during psychosocial stress

Dr Carly J Wood (corresponding author); Lecturer in Exercise and Nutrition Science Department of Life Sciences, University of Westminster, London, W1W6UW; 0207911500 ext. 66415; C.wood@westminster.ac.uk,

Professor Angela Clow; Professor of Psychology, Department of Psychology, University of Westminster, London, W1W6UW; 0207911500 ext. 69061; clowa@ westminster.ac.uk

Professor Frank Hucklebridge; Professor of Psychophysiology, Department of Biomedical Sciences, University of Westminster, London, W1W6UW; 0207911500 ext. 64141;

F.Hucklebridge@westminster.ac.uk

Mr Robin Law; PhD Researcher, Department of Psychology, University of Westminster, London, W1W6UW; 0207911500 ext. 69184; robin.law@my.westminster.ac.uk

Dr Nina Smyth; Senior Lecturer in Psychology, Department of Psychology, University of Westminster, London, W1W6UW; 0207911500 ext. 64425; N.Smyth@westminster.ac.uk

Word Count (excluding abstract, tables, figures and references): 4325 


\begin{abstract}
Background: Evidence linking fitness and decreased psychosocial stress comes from studies of athletes and typically relies upon self-report measures. Furthermore there is little evidence regarding the impact of physical activity (PA) prior to a stressor. The aims of this study were to determine whether fitness and prior PA influence cortisol concentrations during psychosocial stress. Methods: Seventy-five non-athletic participants took part in a submaximal walk prior to the Trier Social Stress Test for Groups (TSST-G). During the walk fitness was assessed using heart rate (HR). A further 89 participants took part in the TSST-G without the walk. Stress responsiveness was assessed using salivary cortisol collected at 10minute intervals on seven occasions. Results: Hierarchical multiple regression revealed that average walking HR accounted for $9 \%$ of the variance in cortisol secretion $(\mathrm{P}=0.016)$; where a higher HR was associated with higher cortisol secretion. Between-subjects ANCOVA revealed that the walking group had a significantly lower cortisol secretion than the nonwalking group ( $\mathrm{P}=0.009)$. Conclusions: These findings indicate that fitter individuals have reduced cortisol secretion during psychosocial stress. They also indicate that prior PA can reduce cortisol concentrations during psychosocial stress and are suggestive of a role of PA in reducing the impact of stress on health.
\end{abstract}

Keywords: fitness, psychosocial stress, physical activity, cortisol, health 


\section{Introduction}

The hypothalamic-pituitary-andrenal (HPA) axis is the body's main physiological stress response system and is typically examined using salivary cortisol. Cortisol plays an essential role in regulating physiological function around the 24-hour light/dark cycle. In healthy individuals, cortisol exhibits a marked circadian rhythm, with peak levels in the morning following awakening and declining levels thereafter (Smyth, Hucklebridge, Thorn, Evans \& Clow, 2013). Both physical (e.g. electric shock) and psychological (e.g public speaking) stressors can activate the HPA axis resulting in cortisol secretion (Dickerson \& Kemeny, 2004). Whilst short-term activation is adaptive and essential for normal functioning, repeated activation can lead to cortisol dysregulation and disrupted circadian patterns; which are related to physical and psychological ill-health (Kyrou \& Tsigos, 2009; McEwen, 2013).

Physical activity (PA) provides well-documented health benefits and is a remedy for many stress related illnesses (Klaperski, von Dawans, Heinrichs \& Fuchs, 2013; 2014; Pasco et al., 2011; Reed \& Buck, 2009; Thompson et al, 2012; Warburton, Nicol \& Bredin, 2006). The cross-stressor adaptation hypothesis suggests that regular PA leads to biological adaptations which contribute to reduced physiological reactions to psychosocial stress (Klaperski et al., 2014). This is because PA itself is considered a stressor and therefore activates the HPA axis. When repeatedly activated in this way the neuroendocrine system response is attenuated resulting in reduced cortisol levels at the same relative exercise intensity (Childs \& De Wit, 2014; Hackney, 2006). It is possible that this attenuated response is also carried over to other stressful situations, resulting in an overall reduction in salivary cortisol secretion (Childs \& De Wit, 2014; Hackney, 2006). Research has identified that individuals who are more physically fit have improved cortisol profiles and reduced physiological responses to stressors (Klaperski et al., 2013; 2014; Strahler, Fuchs, Nater \& Klaperski, 2016). Given that 
high stress reactivity and repeated HPA axis activation have been linked with poor health outcomes (Ozbay et al., 2007), the ability to reduce physiological stress is essential. However, research on this topic to date has typically investigated athletic populations and asssessed physical fitness via self-report measures (Klaperski et al., 2014; Strahler et al., 2016). Whilst determination of $\mathrm{VO}_{2} \max$ is the gold standard for assessing physical fitness, this procedure is time-consuming, maximal and invasive. Heart rate during sub-maximal activities such as walking is negatively correlated with directly measured VO2max (Carter, Banister \& Blaber, 2003; Kilne et al., 1987; Larsen et al., 2002; Zwiren, Freedson, Ward, Wilke \& Rippe, 1991); indicating that heart rate can be used as a reliable physiological marker of fitness, allowing for simple and non-invasive fitness estimation.

Within the literature it is also suggested that regular PA and everyday life activities may be a major confounder in psychosocial stress research in non-athletic groups (Stahler et al., 2016). Furthermore, evidence from a recent meta-analysis indicates that an acute bout of exercise can reduce blood pressure and heart rate responses to a psychosocial stressor (Hamer, Taylor \& Steptoe, 2006). However, there is a lack of research examining the impact of an acute bout of PA on salivary cortisol responses to a stressor. It is suggested that the neuroendocrine response to an acute bout of PA is transient in nature and that during recovery hormonal levels return to baseline or slightly below, very rapidly (Hackney, 2006). However, it is unclear what impact this might have on the cortisol response to a subsequent stressor.

The primary aim of this study was therefore to determine whether physiological fitness influences cortisol concentrations during a group psychosocial stressor. The secondary aim was to examine whether cortisol concentrations during a group psychosocial stressor are influenced by prior participation in PA . 


\section{Method}

\section{Participants}

A total of 164 participants were recruited to take part in the study, all of whom were psychology students. Seventy five of these participants took part in a standardised walk prior to a psychosocial stress test. Eighty nine participants took part in the psychosocial stress testing without the prior walking period. The non-walking group comprised two sub-groups: eleven participants (non-walking group 1) were tested in parallel to the walking group whilst data for 78 participants were derived from identical research procedures (non-walking group 2) (Smyth et al., 2015). All participants were non-athletic; meaning that they did not compete in any sporting activities at a national level or above. Data collection for all participants took place between January and March.

In order to control for gender differences in cortisol responses all participants were female (mean \pm SD age: $20.1 \pm 3$.4years). Exclusion criteria included steroid medication, any illness preventing participation in PA and history of psychiatric illness; all of which are known to influence cortisol secretion (See Smyth et al., 2013). As cortisol secretion is also influenced by the menstrual cycle, the number of days since the last period was recorded alongside measures of oral contraceptive use, height, weight and smoking status (Smyth et al., 2013; 2015). The study was approved by the University Ethics committee. Participants did not receive financial incentives for participation but did receive course credits.

\section{The Walk}

Participant fitness $(n=75)$ was assessed during a thirty minute moderate intensity standardised group walk. The walk took place immediately prior to the preparatory period for the psychosocial stressor (30 minutes prior to the onset of the stress test) and was led by a 
researcher experienced in conducting PA interventions (Figure 1). The walk started and ended at the University site and involved walking in a circular route through urban areas and a local urban park. The walks were of a set distance ( 1.9 miles) and lasted between 30.7 minutes and 34.3 minutes (average 31.9 minutes); with approximately two thirds of the walk being spent in the urban park. The walks were designed to be reflective of an everyday walk in an urban area during which individuals might typically visit a nearby urban greenspace and attempt to meet one of the five days per week in which they are recommended to perform 30 minutes of moderate intensity PA (Townsend, Wickramasinghe, Williams, Bhatnagar \& Rayner, 2015). Heart rate was monitored throughout the walks using FirstBeat Bodyguard 2 heart rate monitors. The monitors were attached to participants using ECG electrodes and provided a measure of average heart rate during the walk. Fitter individuals have lower heart rates at submaximal exercise intensities (Carter et al., 2003); thus those participants with lower average heart rate were deemed to have a greater level of fitness.

\section{Psychosocial stress Test}

All participants took part in the Trier Social Stress Test for Groups (TSST-G) (von Dawans, Kirscbaum \& Heinrichs, 2011) which has been adapted from the Trier Social Stress Test (TSST) (Kirschbaum, Pirke \& Hellhammer, 1993). Experimental procedures for the current study were identical to those previously used by the authors (Smyth et al., 2015). Participants were invited to attend a test session at the University on a weekday lunchtime. In the walking group participants arrived at either $12.30 \mathrm{pm}$ or $14.00 \mathrm{pm}$ for the walking preparation and walk itself. The non-walking group 1 also arrived at this time and were subsequently asked to sit in a room during the period in which the walking group were walking. Following the walking and sitting periods participants went straight into the TSST-G briefing, which took place at either $1.30 \mathrm{pm}$ or $3.00 \mathrm{pm}$ (see Figure 1). The non-walking group 2 arrived in time for the 
TSST-G briefing, which started at either $1.00 \mathrm{pm}, 2.00 \mathrm{pm}$ or $3.00 \mathrm{pm}$ and followed the same procedure and timeline as shown in Figure 1. In line with best practice guidelines testing commenced in the afternoon to control for changes in basal cortisol secretion in the morning and following the post-prandial period (Dockray, Susman \& Dorn, 2009; Smyth et al., 2013). Participants were asked to refrain from food, caffeine and smoking 30 minutes prior to the research session. The non-walking participants were also asked to refrain from exercise during this time.

The TSST-G comprises uncontrollability and socio-evaluative threat known to reliably activate the HPA axis (Dockray \& Kemeny, 2004; Smyth et al., 2015). The TSST-G included three main phases: i) the group preparatory period (30 minutes); ii) the group stress task period (22 minutes) and; iii) a group resting and debriefing period (40 minutes).

During the preparatory period groups of up to six participants assembled in a room where they were seated around a table. The group sizes ranged from four to six, with $68 \%$ of participants being in a group of six, $21 \%$ in a group of five and $11 \%$ in a group of four. In the walking groups, these were also the same participants who had walked together. Following informed consent, participants completed demographic questions and noted the date of their last menstruation. Participants then received a sticker with a number between one and six for identification and random selection during the TSST-G. Following this, participants were then introduced to the saliva sampling method (see below). Finally, participants were given 10-minutes of quiet time to prepare notes for a mock job interview where they were asked to prepare a 2-minute speech as if applying for a job and to introduce themselves to the committee. At the end of this preparation period participants provided their first saliva sample. 
During the TSST-G participants were moved into an additional room and were instructed to stand in a straight line in front of a seated committee consisting of one man and one woman. The committee were wearing white laboratory coats. There were also two video cameras pointing at the participants. A committee member called the number of each participant in turn in a random order to make their two-minute speech; all participants were present in the room for the duration of the task. Standard responses were given by the committee when participants ended their speech early ("you still have time remaining please continue"). On completion of the speeches (12 minutes) the second saliva sample was collected. The committee then asked the participants, in the same order, to serially subtract 17 from a given number as fast and accurately as possible for 80 seconds. Each participant received a new starting number to avoid learning effects. The starting numbers provided were 4878,4862 , 4892, 4856, 4884 and 4871; with each participant in each TSST-G having a different number. If participants failed in the subtraction task, standard responses were employed ("you have made a mistake please start again from the number..."). Following the arithmetic task saliva sample three was collected. Immediately after completion of the TSST-G participants returned to the preparation room. Participants provided saliva samples every 10 minutes up to 40 minutes (samples 4-7). During the recovery saliva collection period they were also debriefed on the study. A timeline outling the experimental protocol is provided in Figure 1.

\section{Saliva sampling}

Cortisol was measured in saliva samples collected through use of salivettes (Sarstedt Ltd., Leceister, UK). The salivettes were push caps with swabs made of polypropylene; these salivettes were only suitable for analysis of salivary cortisol. In all participants samples were collected at baseline (immediately before the TSST-G at 0 mins); immediately after the public speaking task (12 mins), after the mental arithmetic task (22 mins); and every 10 mins 
up to 62 minutes. All saliva samples were frozen at $-20^{\circ} \mathrm{C}$ until assayed. Samples were thawed and then centrifuged for 10minutes at a speed of $3500 \mathrm{rpm}$. Cortisol concentrations were determined in duplicates at the Psychophysiology and Stress Research Groups' laboratory at the University and were established by enzyme-linked-immunosorbent assaying, developed by Salimetrics LLC (State College, PA). The standard range in the assay was 0.33$82.77 \mathrm{nmol} / \mathrm{l}$. Intra- and inter-assay variations were both $<10 \%$.

\section{Statistical analysis}

Cortisol data was skewed and a square root transformation was therefore applied to normalize the distributions prior to statistical analyses (von Dawans et al., 2011). However, the raw cortisol concentrations are displayed in both tables and figures. Cortisol composites were calculated for overall cortisol secretion as "area under curve with respect to ground" $\left(\mathrm{AUC}_{\mathrm{g}}\right)$ (Pruessner, Kirschbaum, Meinlschmid \& Hellhammer, 2003). Prior to the main analysis, between subjects ANCOVA compared the effect of both test time and test group size on $\mathrm{AUC}_{\mathrm{g}}$ whilst controlling for age, BMI, oral contraceptive use, menstrual phase and smoking status.

One-way within subjects analysis of variance (ANOVA) was conducted to examine differences in cortisol secretion over time for the walking group. Within-subjects contrasts were used to assess the pattern of cortisol secretion. Hierarchical multiple regression was used to assess the impact of fitness (using average walking heart rate as a continuous variable) on cortisol concentrations $\left(\mathrm{AUC}_{\mathrm{g}}\right)$ whilst controlling for participant age, BMI, oral contraceptive use, menstrual phase and smoking status; all of which are known to affect cortisol values. 
The walking group data was also split into two groups; a 'fit' and 'unfit' group. The walk was designed to be of a moderate intensity. Heart rate during moderate intensity PA is estimated to be between $55-70 \%$ of maximum heart rate (Norton, Norton \& Sadgrave, 2010); the fit group was therefore defined as participants whose average heart rate during the walk was less than or equal to $70 \%$ of maximum heart rate. Maximum heart rate was calculated using the well-known prediction equation of 220-age (Norton, Norton \& Sadgrave, 2010), and using the average age of the walking group (19.3years). Thus, the predicted maximum heart rate was $201 \mathrm{bpm}$; with $70 \%$ of maximum heart rate being equivalent to $141 \mathrm{bpm}$. The unfit group were participants whose average heart rate during the walk was greater than $141 \mathrm{bpm}$. A between subjects ANCOVA compared the effect of fitness group on $\mathrm{AUC}_{\mathrm{g}}$ whilst controlling for age, BMI, oral contraceptive use, menstrual phase and smoking status.

In order to ensure there were no differences between the two non-walking groups before combining data, independent t-tests were used to compare age and BMI; whilst Chi-square tests were used to compare smoking status, menstrual phase and oral contraceptive use. Differences in $\mathrm{AUC}_{\mathrm{g}}$ between the non-walking groups were also assessed using one-way between subjects ANCOVA whilst controlling for variables known to influence cortisol values.

Following combination of the data, independent t-tests were also used to compare age and BMI in the walking and non-walking groups; whilst Chi-square tests were used to compare smoking status, menstrual phase and oral contraceptive use. One-way within subjects ANOVA was also conducted to examine differences in cortisol over time for all groups. Within-subjects contrasts were used to assess the pattern of cortisol secretion. Differences in $\mathrm{AUC}_{\mathrm{g}}$ between the two groups were also assessed using one-way between subjects 
ANCOVA, whilst controlling for participants age, BMI, oral contraceptive use, menstrual phase and smoking status.

\section{Results}

\section{Comparison of group size and test time}

Between subjects ANCOVA revealed no significant differences between the participants $\mathrm{AUC}_{\mathrm{g}}$ as a result of their TSST-G group size after controlling for variables known to affect cortisol responses $\left(\mathrm{P}=0.208 ; \mathrm{n}_{\mathrm{p}}^{2}=.022\right)$. Between subjects ANCOVA also revealed no significant differences between the participants $\mathrm{AUC}_{\mathrm{g}}$ due to their TSST-G time test $\left(\mathrm{P}=0.133 ; \mathrm{n}_{\mathrm{p}}^{2}=.040\right)$. These variables were not therefore controlled for in the analysis.

\section{Walking group Assessment}

The TSST-G induced an overall cortisol response $\left[\mathrm{F}(6,396)=10.18 ; \mathrm{P}<0.001, \mathrm{n}_{\mathrm{p}}^{2}=.134\right]$ that did not distinguish the 'fit' and 'unfit' groups. Within-subjects contrasts revealed a significant quadratic effect $\left[\mathrm{F}(1,66)=22.26 ; \mathrm{P}<0.001, \mathrm{n}^{2}{ }_{\mathrm{p}}=.252\right]$. Participants experienced an increase in cortisol levels from baseline, with cortisol peaking at sample 4 (ten minutes post the TSST-G) and declining thereafter.

Hierarchical multiple regression revealed that the average heart rate during the walk accounted for $9 \%(\mathrm{R}$ square change $=0.90)$ of the variance in $\mathrm{AUC}_{\mathrm{g}}$ after controlling for age, BMI, oral contraceptive use, menstrual phase and smoking status $[\mathrm{F}(1,58)=6.16 ; \mathrm{P}=0.016)$. None of the control variables made a significant contribution to the variance in $\mathrm{AUC}_{\mathrm{g}}$. Average heart rate made a unique, significant contribution (beta $=0.31 ; \mathrm{P}=0.016$ ); in which a higher heart rate was associated with a higher $\mathrm{AUC}_{\mathrm{g}}$. 
Between subjects ANCOVA revealed a significant difference between the 'fit' and 'unfit' groups in their $\mathrm{AUC}_{\mathrm{g}}$ after controlling for variables known to affect cortisol responses $\left[\mathrm{F}(1,52)=5.13 ; \mathrm{P}=0.033 ; \mathrm{n}^{2} \mathrm{p}=.09\right]$. The 'fit' group had a lower overall AUC $\mathrm{g}_{\mathrm{g}}$ compared to the 'unfit' group (Figure 2).

\section{Comparison of walking and non-walking groups}

Demographic data

Non-walking groups. Independent t-tests revealed no significant differences between the two cohorts of non-walking participants in terms of age $\left(\mathrm{P}=0.340 ; \mathrm{n}^{2} \mathrm{p}=.011\right)$ or $\mathrm{BMI}(\mathrm{P}=0.527$; $\left.\mathrm{n}^{2} \mathrm{p}=.005\right)$. A chi-square test also revealed no significant differences in smoking status $(\mathrm{P}=0.261 ; \mathrm{V}=.119)$, menstrual phase $(\mathrm{P}=0.657 \mathrm{~V}=.047)$ or oral contraceptive use $(\mathrm{P}=0.591$; $V=.057)$. The majority of participants in both groups were non-smokers, in the luteal phase of their menstrual cycle and did not use oral contraceptives (Table 1).

Between subjects ANCOVA also revealed no significant differences between the two cohorts in their $\mathrm{AUC}_{\mathrm{g}}$ after controlling for variables known to affect cortisol responses $\left(\mathrm{P}=0.979 ; \mathrm{n}_{\mathrm{p}}^{2}=\right.$ $.000)$. The non-walking group data was therefore combined.

Walking and non-walking groups. There were no differences between the walking and nonwalking group in terms of age $\left(\mathrm{P}=0.990 ; \mathrm{n}^{2}{ }_{\mathrm{p}}=.000\right)$, BMI $\left(\mathrm{P}=0.268 ; \mathrm{n}^{2}{ }_{\mathrm{p}}=.008\right)$, smoking status $(\mathrm{P}=0.334 ; \mathrm{V}=0.076)$ or menstrual phase $(\mathrm{P}=0.074 ; \mathrm{V}=0.143)$. A chi-square test revealed a significant difference between the two groups in terms of oral contraceptive use $\left(X^{2}=19.76\right.$; $\mathrm{P}<0.001 ; \mathrm{V}=0.348$ ). A greater proportion of participants in the walking group reported using oral contraceptives (Table 2). 
Response to the TSST-G. The TSST-G induced an overall cortisol response $[\mathrm{F}(6,954)=$ 21.84; $\left.\mathrm{P}<0.001, \mathrm{n}_{\mathrm{p}}^{2}=.121\right]$ that did not differ between the walking and non walking groups. Within-subjects contrasts revealed a significant quadratic effect $[\mathrm{F}(1,159)=63.08 ; \mathrm{P}<0.001$, $\left.\mathrm{n}_{\mathrm{p}}^{2}=.284\right]$. Participants experienced an increase in cortisol levels from baseline, with cortisol peaking at sample 4 (ten minutes post the TSST-G) and declining thereafter

Between subjects ANCOVA also revealed a significant difference in the $\mathrm{AUC}_{\mathrm{g}}$ between the walking and non-walking groups after controlling for age, BMI, oral contraceptive use, menstrual phase and smoking status $\left[\mathrm{F}(1,143)=7.06 ; \mathrm{P}=0.009 ; \mathrm{n}_{\mathrm{p}}^{2}=.047\right]$. The walking group had a lower $\mathrm{AUC}_{\mathrm{g}}$ than the non-walking group (Figure 3).

\section{Discussion}

The findings of this study indicate that there was a relationship between physical fitness and cortisol secretion in non-athletic healthy individuals: individuals who had a lower average heart rate during a submaximal walking activity (and therefore an enhanced level of fitness) had lower cortisol secretion during a psychosocial stressor. These findings are in line with previous research demonstrating a relationship between self-reported fitness and physiological stress responses; with blunted cortisol responses in fitter individuals (Klaperski et al., 2013, 2014; Rimmele et al., 2007; 2009; Strahler et al., 2016). However, the majority of these studies used self-reported measures of stress; or compared stress responses in highly trained and sedentary individuals (Strahler et al., 2016). This is the first known study to examine the relationship between stress responses and fitness measured using physiological measures in an entirely non-althletic group and indicates that greater fitness in a non-athletic population may play an important role in physiological stress regulation. Although there were no differences in cortisol responses to the TSST-G between the two groups, overall cortisol 
output was lower in the 'fitter' group. Increased cortisol secretion has been associated with ill-health (see Smyth et al., 2013); this finding therefore indicates that fitness in a non-athletic population could play a key role in reducing the negative impact of stress on health.

The secondary finding of this study was that individuals' who participate in PA immediately prior to a stressor have a significantly lower cortisol secretion. Whilst the TSST induced an overall cortisol response in both the walking and non-walking groups; participants in the walking group had significantly lower levels of cortisol throughout the sampling period.

The reduced cortisol concentrations in the walking group are likely to be a direct result of the PA. There is a growing and convincing body of evidence to suggest that participation in PA can reduce physiological stress regulation and improve overall health and wellbeing (Klaperski et al., 2013, 2014; Pasco et al., 2011; Reed \& Buck 2009; Thompson et al., 2012; Warburton et al., 2006). However, this evidence has been based upon overall levels of PA as opposed to the impact of participation in PA immediately prior to a stressor. Individuals in the walking group were of varied fitness with some participants anedcotally reporting that they did very little PA in their everyday lives. These findings suggest that a manipulation of PA on any given day might influence subsequent cortisol secretion; meaning that an individual who does not regularly take part in PA can modify their stress regulation following participation in one PA session. In addition, these results concur with the suggestion that everyday PA is likely to a be a major confounder in stress research (Strahler et al., 2016) and indicate that a one-off walk of short duration can significantly reduce salivary cortisol secretion. This finding suggests that PA could be implemented as a strategy for dealing with anticipated stresses. Given that high cortisol secretion and consequent disrupted cortisol levels are related to physical and psychological ill-health (see Smyth et al., 2013); this might 
be an important strategy for minimising stress and could therefore result in improved public health through a reduction in stress related ill-health.

It could also be speculated that the contact with nature experienced during the park section of the walk (defined as Green Exercise (GE): 'physical activity whilst exposed to nature') contributed to the reduced cortisol levels. There is an increasing body of evidence to suggest that contact with natural environments, including urban green spaces; can significantly reduce physiological stress via reductions in salivary cortisol (Lee et al., 2011; Roe et al., 2013; Thompson et al., 2013). In fact, Ulrich's psycholevolutionary stress reduction model suggests that there is a direct impact of nature on both body and mind and that this impact occurs via psychoneuroendocrine mechanisms including the HPA axis (Ulrich, 1983; Ulrich et al., 1991). In addition GE has been demonstrated to provide additive benefits for health and wellbeing above exercise in other environments, including reductions in self-reported stress (Barton \& Pretty, 2010; Barton, Griffin \& Pretty, 2012; Bowler, Buyung-Ali, Knight \& Pullin, 2010; Klaperski et al., 2014; Pretty et al., 2007; Thompson et al., 2012; Thompson Coon et al., 2011; Wood, Pretty \& Griffin, 2015). However; further research is required to confirm whether a nature-based walk is more effective at reducing physiological stress than a walk in a non-green area.

The results of the study are limited to healthy young females. It would therefore be interesting to examine the impact of fitness and prior PA on salivary cortisol concentrations during a stressor in a male population and indeed older and less healthy groups. The use of data from two sets of non-walking participants is a limitation. However, a sub-group of nonwalking participants were studied alongside the walking group and found to be not different to the previously collected non-walking group, which legitimised the data merger. There were 
also no differences between participants who took part in the TSST-G at different times or in different group sizes. Furthermore, all assays were conducted in the same laboratory. It would have also been beneficial to have controlled the activities that the non-walking groups took part in during the 30 minutes prior to the TSST testing session to ensure that their experiences were identicial. The walking participants walked in the same groups in which they took part in the TSST-G and may therefore felt more connected and supported during the TSST-G than the non-walking groups. This support might have influenced overall cortisol responses. However, the non-walking group 1 did have the same opportnity for social bonding by sitting in a room together for 30 minutes prior to the TSST briefing and the test itself, providing a control for this possibility.

The method of assessing fitness could also be considered as a limitation. It would have been more accurate to assess fitness through use of a $\mathrm{VO}_{2} \max$ or submaximal fitness test as opposed to using heart rate during submaximal exercise as a predictor of fitness. However, it is likely that these testing procedures may have mitigated against participant recruitment and adherence; particularly the use of a $\mathrm{VO}_{2} \max$ test which is an instrusive and exhaustive test. Furthermore, heart rate during submaximal PA is accepted as a good marker of fitness (Carter et al., 2003).

The findings of this study identify a clear need for further research in the area of PA and psychosocial stress. Whilst, there is evidence to suggest that individuals who are more active and fit have reduced physiological responses to stress (Klaperski et al., 2014; Strahler et al., 2016), and that long term exercise interventions can reduce stress reactivity (Klaperski et al., 2013); research has typically involved small sample sizes and has not considered the potential impact of a bout of PA immediately prior to a stressor. In the current study the PA took place 
30 minutes prior to onset of the stressor. It is possible that this timing could play a significant role in the PA-stress relatonship, as could the duration and intensity of the exercise (Childs \& De Wit, 2014; Hackney, 2006). However, there is little research in this area and these relationships therefore need exploring further in order to optimise health interventions. Research has also not considered the use of natural environments in reducing stress. It would therefore be useful to determine whether there are differences in cortisol secretion not only in individuals who are physically active but also who regularly use green space for both leisure and PA. There is also a need for research to examine whether GE interventions can be used as a tool for modifying stress responses and whether their impact is greater than traditional exercise interventions. If this is the case, this could have important implications for stress coping and public health.

In summary, the findings of this study indicate that non-athletic young females with greater fitness, as well as prior moderate intensity PA for 30 minutes, are both associated with lower salivary cortisol concentrations during psychosocial stress. These differences remain significant when controlling for age, smoking status, BMI, oral contraceptives and menstrual phase. These results are suggestive of a potential role of fitness in reducing physiological stress and indicate that PA could be used as a tool for modifying susceptibility to stressrelated ill health.

\section{Funding:}

This was work was Supported by the Bial Foundation under grant number 72/12 and the University of Westminster start-up funding. 


\section{References}

Barton, J., Griffin, M., \& Pretty, J. (2012). Exercise-, nature- and socially interactive-based initiatives improve mood and self-esteem in the clinical population. Perspectives in Public Health, 132, 89-96. doi: 10.1177/1757913910393862

Barton, J., \& Pretty, J. (2010). What is the Best Dose of Nature and Green Exercise for Improving Mental Health? A Multi-Study Analysis. Environmental Science and Technology, 44, 3947-55. doi: 10.1021/es903183r.

Bowler, D.E., Buyung-Ali, L.M., Knight, T.M., \& Pullin, A.S. (2010). A systematic review of evidence for the added benefits to health of exposure to natural environments. BMC Public Health, 10, 456-466. doi: 10.1186/1471-2458-10-456

Carter, J.B., Banister, E.W., \& Blaber, A.P. (2003) Effect of endurance exercise on autonomic control of heart rate. Sports Medicine, 33, 33-46. doi: 10.2165/00007256200333010-00003

Childs, E., \& de Wit, H. (2014). Regular exercise is assoiciated with emotional resilience to acute stress in health adults. Frontiers in Physiology, 5: 1-10. doi:10.3889/fphys.2014.00161 Dickerson, S.S., \& Kemeny, M.E. (2004). Acute stressors and cortisol responses: a theoretical integration and synthesis of laboratory research. Psychological Bulletin, 130, 355391. doi: 10.1037/0033-2909.130.3.355

Dockray, S., Susman, E.J., \& Dorn, L.D. (2009). Depression, Cortisol Reactivity, and Obesity in Childhood and Adolescence. Journal of Adolescent Health, 45, 344-50. doi: 10.1016/j.jadohealth.2009.06.014

Hackney, A.C. (2006). Stress and the neuroendocrine system: the role of exercise as a stressor and modifier of stress. Expert Review of Endocrinology and Metabolism, 1: 783-792. doi: 10.1586/17446651.1.6.783 
Hamer, M., Taylor, A., \& Steptoe, A. (2006). The effect of acute aerobic exercise on stress related blood pressure responses: A systematic review and meta-analysis. Biological Psychology, 71, 183-190. Doi: 10.1016j.biopsycho.2005.04.004

Kilne, G.M., Porcari, J.P., Hintermeister, R., Freedson, P.S., Ward, A., McCarron, R.F., ... Rippe, J.M. (1987). Estimation of $\mathrm{VO}_{2}$ max from a one-mile track walk, gender, age and body weight. Medicine and Science in Sports and Exercise, 19, 253-259. Retrieved from: https://www.ncbi.nlm.nih.gov/pubmed/3600239 Kirschbaum, C., Pirke, K.M., \& Hellhammer, D.H. (1993). The 'Trier Social Stress Test'-a tool for investigating psychobiological stress responses in a laboratory setting.

Neuropsychobiolog, 28, 76-81. Retrieved from:

\section{https://www.karger.com/Article/Abstract/119004}

Klaperski, S., von Dawans, B., Heinrichs, M., \& Fuchs, R. (2013). Does the level of physical exercise affect physiological and psychological responses to psychosocial stress in women? Psychology of Sport and Exercise, 14, 266-74. doi: 10.1016/j.psysport.2012.11.003 Klaperski, S., von Dawans, B., Heinrichs, M., \& Fuchs, R. (2014). Effects of a 12-week endurance training program on the physiological response to psychosocial stress in men: a randomized controlled trial. Journal of Behavioural Medicine, 37, 1118-33. doi:10.1007/s10865-014-9562-9

Kyrou, I., \& Tsigos, C. (2009) Stress hormones: physiological stress and regulation of metabolism. Current Opinion in Pharmocology; 9, 787-793. doi: 10.1016/j.coph.2009.08.007 McEwen, B.S. (2013). Hormones and the Social Brain. Science, 339, 279-80. doi:10.1126/science.1233713. Larsen, G.E., George, J.D., Alexander, J.L, Fellingham, G.W., Aldana, S.G., \& Parcell, A.C. (2002). Prediction of maximum oxygen consumpton from walking, jogging, or running. Research Quarterly in Exercise and Sport, 73, 66-72. doi: 10.1080/02701367.2002.10608993 
Lee, J., Park, B.J., Tsunetsugu, Y., Ohira, T., Kagawa, T., \& Miyazaki, Y. (2011). Effect of forest bathing on physiological and psychological responses in young Japanese male subjects. Public Health, 125, 93-100. doi: 10.1016/j.puhe.2010.09.005

Norton, K., Norton, L., \& Sadgrove, D. (2010). Position statement on physical activity and exercise intensity terminology. Journal of Science and Medicine in Sport, 13, 496-502. doi:10.1016/j.jsams.2009.09.008

Ozbay, F., Johnson, D.C., Dimoulas, E., Morgan, C.A., Charney, D., \& Southwick, S. (2007). Social Support and Resilience to Stress: From Neurobiology to Clinical Practice. Psychiatry, 4, 35-40. Retrieved from: https://www.ncbi.nlm.nih.gov/pmc/articles/PMC2921311/ Pasco, J.A., Jacka, F.N., Williams, L.J., Brennan, S.L., Leslie, E., \& Berk, M. (2011). Don't worry, be active: positive affect and habitual physical activity. The Australian and New Zealand Journal of Psychiatry, 45, 1047-52. doi: 10.3109/00048674.2011.621063 Pretty, J., Peacock, J., Hine, R., Sellens, M., South, N., \& Griffin, M. (2007). Green exercise in the UK countryside: Effects on health and psychological well-being, and implications for policy and planning. Journal of Environmental Planning and Management, 50, 211-31. doi:10.1080/09640560601156466

Pruessner, J.C., Kirschbaum, C., Meinlschmid, G., \& Hellhammer, D.H. (2003). Two formulas for computation of the area under the curve represent measures of total hormone concentration versus time dependent change. Psychoneuroendocrinology, 28, 916-931. doi:10.1016/s0306-453000108-7

Reed, J., \& Buck, S. (2009). The effect of regular aerobic exercise on positive-activated affect: A meta-analysis. Psychology of Sport and Exercise, 10, 581-94. doi:10.1016/j.psychsport.2009.05.009 
Rimmele, U., Seiler, R., Marti, B., Wirtz, P.H., Ehlert, U., \& Heinrichs, M. (2009). The level of physical activity affects adrenal and cardiovascular reactivity to psychosocial stress. Psychoneuroendocrinology, 34, 190-198. doi: 10.1016/j.psyneuen.2008.08.023 Rimmele, U., Zellweger, B.C., Marti, B., Seiler, R., Mohiyeddini, C., Ehlert, U., ... Heinrichs, M. (2007). Trained men show lower cortisol, heart rate and psychological responses to psychosocial stress compared with untrained men. Psychoneuroendocrinology, 32, 627-635. doi: 1016/j.psyneuen.2007.04.005

Roe, J.J., Thompson, C.W., Aspinall, P.A., Brewer, J., Duff, E.I., Miller, R., .. Clow, A. (2013). Green Space and Stress: Evidence from Cortisol Measures in Deprived Urban Communities. International Journal of Environmental Research and Public Health, 10, 4086-103. doi: 10.3390/ijerph10094086

Smyth, N., Hucklebridge, F., Thorn, L., Evans, P., \& Clow, A. (2013). Salivary cortisol as a biomarker in social science research. Social Personality Psychology Compass, 7, 605-25. doi:10.111/spc3.12057

Smyth, N., Thorn, L., Oskis, A., Hucklebridge, F., Evans, P., \& Clow, A. (2015). Anxious attachment style predicts an enhanced cortisol response to group psychosocial stress. Stress, 18, 143-8. doi: 10.3109/10253890.2015.1021676

Strahler, J., Fuchs, R., Nater, U.M., \& Klaperski, S. (2016). Impact of physical fitness on salivary stress markers in sedentary to low-active young to middle-aged men. Psychoneuroendocrinology, 68, 14-19. doi: 10.1016/j.psyneuen.2016.02.022 Thompson, C.W., Roe, J., Aspinall, P., Mitchell, R., Clow, A., \& Miller, D. (2012). More green space is linked to less stress in deprived communities: Evidence from salivary cortisol patterns. Landscape and Urban Planning, 105, 221-229.

doi:10.1016/j.landurbplan.2011.12.015 
Thompson Coon, J., Boddy, K., Stein, K., Whear, R., Barton, J., \& Depledge, M.H. (2011). Does participating in physical activity in outdoor natural environments have a greater effect on physical and mental wellbeing than physical activity outdoors? A systematic review. Environmental Science and Technology, 45, 1761-1772. doi:10.1021/es102947t

Townsend, N., Wickramasinghe, K., Williams, J., Bhatnagar, P., \& Rayner, M. (2015). Physical Activity Statistics 2015. London: British Heart Foundation.

Ulrich, R.S. (1983). Aesthetic and Affective Response to Natural Environment. In I. Altman, \& J.F. Wohlwill (Ed.), Behavior and the Natural Environment (pp. 85-125). Boston, MA: Springer US.

Ulrich, R.S., Simons, R.F., Losito, B.D., Fiorito, E., Miles, M.A., \& Zelson, M. (1991). Stress recovery during exposure to natural and urban enviroments. Journal of Environmental Psychology, 11, 201-30. doi: 10.1016/S0272-494480184-7/

von Dawans, B., Kirschbaum, C., \& Heinrichs, M. (2011). The Trier Social Stress Test for Groups (TSST-G): A new research tool for controlled simultaneous social stress exposure in a group format. Psychoneuroendocrinology, 36, 514-22. doi:10.1026/j.psyneuen.2010.08.004 Warburton, D.E.R., Nicol, C.W., \& Bredin, S.S.D. (2006). Health benefits of physical activity: the Evidence. Canadian Medical Association Journal, 174, 801-9.

doi:10.1503/cmaj.051351

Wood, C.J., Pretty, J., \& Griffin, M. (2015). A case-control study of the health and wellbeing benefits of allotment gardening. Journal of Public Health, 38, e336-344.

doi:10.1093/pubmed/fdv146

Zwiren, L.D., Freedson, P.S., Ward, A., Wilke, S., \& Rippe, J.M. (1991). Estimation of $\mathrm{VO}_{2}$ max: A comparative analysis of five exercise tests. Research Quarterly in Exercise and Sport, 62, 73-78. Retrieved from:

http://www.tandfonline.com/doi/abs/10.1080/02701367.1991.10607521 
Tables

Table 1: Demographic information of the two non-walking groups.

Non-walking group $1 \quad$ Non-walking group 2

$$
(\mathbf{n}=11) \quad(\mathbf{n}=78)
$$

\begin{tabular}{c}
\hline Age (years) \\
\hline BMI $\left({\left.\mathrm{Kg} . \mathrm{m}^{2}\right)}^{2}\right.$
\end{tabular}

$19.3 \pm 1.4$

$20.2 \pm 3.1$

Smoker (\%)

$22.3 \pm 3.5$

$21.5 \pm 3.8$

\begin{tabular}{cc}
\hline \multirow{2}{*}{ Smoking status } & Smoker (\%) \\
\cline { 2 - 2 } phase & Non-smoker (\%) \\
\cline { 2 - 2 } Menstrual & Ruteal (\%) \\
\hline Oral & Yest(\%) $(\%)$ \\
contraceptive & \\
\cline { 2 - 2 } use & No (\%) \\
\end{tabular}

27.3

14.1

72.7

85.9

54.5

61.5

45.5

38.5

0

2.6

$\operatorname{AUC}_{g}(\mathbf{n m o l} / \mathbf{l})$

$40.8 \pm 28.1$

$41.1 \pm 28.5$

Note: $\mathrm{BMI}=$ body mass index; $\mathrm{AUC}=$ area under the curve with respect to ground 
Table 2: Demographic information for the walking and non-walking groups.

\begin{tabular}{|c|c|c|c|}
\hline & & Non-walking group $(n=89)$ & Walking group $(n=75)$ \\
\hline \multicolumn{2}{|c|}{ Age (years) } & $20.2 \pm 3.1$ & $19.3 \pm 1.4$ \\
\hline \multicolumn{2}{|c|}{ BMI (Kg.m²) } & $21.5 \pm 3.8$ & $22.3 \pm 3.5$ \\
\hline \multirow[b]{2}{*}{ Smoking status } & Smoker (\%) & 15.7 & 21.6 \\
\hline & $\begin{array}{c}\text { Non-smoker } \\
(\%)\end{array}$ & 84.3 & 78.4 \\
\hline Menstrual & Luteal (\%) & 60.7 & 46.3 \\
\hline phase & $\operatorname{Rest}(\%)$ & 39.3 & 53.7 \\
\hline \multirow{2}{*}{$\begin{array}{c}\text { Oral } \\
\text { contraceptive }\end{array}$} & Yes $(\%)$ & 2.2 & $25.7 *$ \\
\hline & No $(\%)$ & 97.8 & 74.3 \\
\hline
\end{tabular}


Figure Legends

Figure 1. Experimental Timeline

Figure 2. Mean $( \pm$ SEM) salivary free cortisol concentrations (nanomoles per litre) for 'fit' and 'unfit' groups. (A) Immediately before onset of the Trier Social Stress Test for Groups (TSST-G); (B) mid-way through the TSST-G and (C) immediately after the end of the TSST-G

Figure 3. Mean $( \pm$ SEM) salivary free cortisol concentrations (nanomoles per litre) for walking and non-walking groups. (A) Immediately before onset of the Trier Social Stress Test for Groups (TSST-G); (B) mid-way through the TSST-G and (C) immediately after the end of the TSST-G 